Conclusions: The prevalence of the musculoskeletal manifestations is high $(76.4 \%)$ in the hemodialysis population. Musculoskeletal disorders seem to cluster differently according to age and dialysis vintage.

Acknowledgements: Dr Ghada Abi Karam and Dr Fouad Fayad.

Disclosure of Interest: None declared

DOI: 10.1136/annrheumdis-2018-eular.4217

\section{AB1337 CELIAC DISEASE AND RISK OF SARCOIDOSIS: A SYSTEMATIC REVIEW AND META-ANALYSIS}

K. Wijarnpreecha ${ }^{1}$, P. Panjawatanan ${ }^{2}$, P. Lertjitbanjong ${ }^{1}$, J.E. Corral ${ }^{3}$, P. Ungprasert ${ }^{4}{ }^{1}$ Medicine, Bassett medical center, cooperstown, USA; ${ }^{2}$ Biochemistry, Faculty of Medicine, Chiang Mai University, Chiang Mai, Thailand; ${ }^{3}$ Gastroenterology and Hepatology, Mayo Clinic, Jacksonville, USA; ${ }^{4}$ Research and development, Faculty of Medicine Siriraj Hospital, Mahidol University, Bangkok, Thailand

Background: Several epidemiologic studies have demonstrated that patients with celiac disease may be at an increased risk of devleoping sarcoidosis. However, the data on this risk remain inconsistent.

Objectives: To compare the risk of developing sarcoidosis between patients with celiac disease and individuals without celiac disease.

Methods: A literature review was performed using MEDLINE and EMBASE database from inception to December 2017. Studies that compared the risk of sarcoidosis among patients with celiac disease versus those without celiac disease were included. The inclusion criteria were as follows: ${ }^{1}$ case-control, cross-sectional or cohort studies that investigated the risk of sarcoidosis among patients with celiac disease compared with individuals without celiac disease and ${ }^{2}$ odds ratios $(\mathrm{OR})$, relative risks $(\mathrm{RR})$, hazard ratios $(\mathrm{HR})$ or standardised incidence ratios (SIR) with $95 \%$ confidence intervals $(\mathrm{Cl})$ or sufficient raw data to calculate these ratios were provided. Study eligibility was independently determined by 3 investigators. Differences in the determination of study eligibility were resolved by mutual consensus. Pooled odds ratio $(\mathrm{OR})$ and $95 \%$ confidence interval $(\mathrm{Cl})$ were calculated using a random-effect, generic inverse variance method of DerSimonian and Laird.

Results: Of 375 retrieved studies, 4 studies ( 2 case-control studies and 2 cohort studies) with 693639 participants met our eligibility criteria and were included in analysis. We found a higher risk of sarcoidosis among patients with celiac disease compared with individuals without celiac disease with the pooled OR of 7.16 (95\% $\mathrm{Cl}, 1.48-34.56)$. The statistical heterogeneity of this study was high $\left(\mathrm{I}^{2=} 95 \%\right)$. Funnel plot was relatively symmetric and did not suggest the presence of publication bias in favour of positive studies.

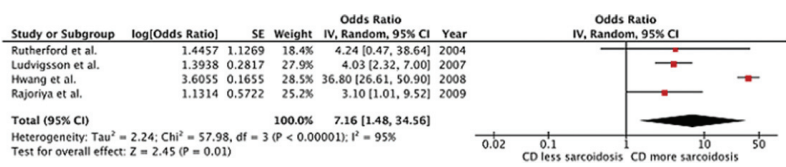

Abstract AB1337 - Figure 1. Forest plot of this meta-analysis

Conclusions: This systematic review and meta-analysis found a significantly higher risk of sarcoidosis among patients with celiac disease.

Disclosure of Interest: None declared

DOI: 10.1136/annrheumdis-2018-eular.4682

\section{AB1338 COHORT OF PARAGUAYAN PATIENTS WITH EARLY ONSET ARTHRITIS}

P.D. Delgadillo Benitez ${ }^{1}$, S. Cabrera Villalba ${ }^{1}$, G. Ávila Pedretti ${ }^{1}$, I. Acosta Colmán $^{2}$, P. Melgarejo ${ }^{1}$, M. Franco ${ }^{1}$, G. Elizaur ${ }^{1}$, J. Mazzoleni $^{1}$, A. Ramagli ${ }^{3}$, P. de Abreu ${ }^{4}$. ${ }^{1}$ Servicio de Reumatología, Instituto de Previsión Social; ${ }^{2}$ Reumatología Hospital de Clínicas, Asunción, Paraguay; ${ }^{3}$ Reumatología, Instituto Nacional de Reumatología, Montevideo, Uruguay, ${ }^{4}$ Reumatología, Sociedad Paraguaya de Reumatología, Asunción, Paraguay

Background: The PANLAR-EOA (early-onset-arthritis) project includes panamericanrheumatologists to determine regional characteristics of patients with early onset arthritis.

Objectives: To describe the cohort of Paraguayan patients included in PANLAREOA project.

Methods: Longitudinal, prospective, multicentricstudy. Patients were included according to the PANLAR-EOA project and registered in REPANARC(www.panlareoa.org) database. At baseline and annual visits, a large number of demographic, clinical and analytical variables were recorded. Quantitative variables were characterised by their means and standard deviations, while the qualitative variables were characterised according to the percentage of patients. The comparison of epidemiological and clinical variables was performed using the chisquared test and the Wilcoxon test respectively for qualitative and quantitative variables, respectively.

Results: 136 patients with early onset arthritis were included, out of which 88 completed the 12 months follow up and 58 the 24 months one. In these, $86 \%$ were female with a median age of $43.9 \pm 13.2$ years. The most frequent race was mes tiza in $80.1 \%$. According to GRAFFAR index, middle class was the predominant social stratum $(9.8 \pm 3.1)$. The average number of years of schooling was 12.8 \pm 3 .8. Polyarticular onset was registeredin $61 \%$ patients. During follow-up, $43.1 \%$ had positive rheumatoid factor and $56.5 \%$ positive anti-CCP. The diagnostic delay was $3.9 \pm 3.0$ months. Initially, $63.2 \%(86 / 136)$ were diagnosed with rheumatoid arthritis (RA) and $36.8 \%(50 / 136)$ with undifferentiated arthritis (UA). The most frequent treatment was methotrexate $(85.3 \%, 90.9 \%$, and $89.3 \%$ at baseline, 1 and 2 years of follow-up respectively). During follow-up, a significant diagnostic change was observed in patients with UA ( $p=0.004, \mathrm{OR}=2.9[95 \% \mathrm{Cl}, 1.4-6.5])$. The variables associated with RA diagnosis werepresence of anti-CCP $(p=0.000, O R=15.8$ [95\%Cl,5.4-51.1]), rheumatoid factor ( $p=0.000$, OR=9.2 [95\% $\mathrm{Cl}, 3.4-27.0])$, smoking $(p=0.032, O R=8.8[95 \% C l, 1.1-404.7])$, high body mass index $(p=0.041$ $\mathrm{OR}=1.94[95 \% \mathrm{Cl},-0.2-4.1])$ and high activity measured by the DAS28 index $(p=0.01)$. After one year of follow-up there was a significant decrease in disease activity according to DAS $28(\mathrm{p}=2.2 \mathrm{e}-09[95 \% \mathrm{Cl},-1.5,-0.9])$, SDAI $(\mathrm{p}=1.2 \mathrm{e}-11$ [95\% Cl, 18.2,-11.2]) and HAQ $(p=7.2 e-08[95 \% \mathrm{Cl},-0.7,-0.4])$. Similar results were found at the 2 nd year of follow-up, DAS28 ( $\mathrm{p}=8.8 \mathrm{e}-06[95 \% \mathrm{Cl},-1.6,-0.7])$, SDAI ( $p=2.1$ e- $07[95 \% \mathrm{Cl},-20.0,-10.3])$ and HAQ $(p=3.4 e-08[95 \% \mathrm{Cl},-0.9,-$ $0.5])$.

Conclusions: In this cohort of early onset arthritis, diagnostic delay was lower than that observed in other series and the rate of change from diagnosis of UA to RA was statistically significant during the first year of follow-up. A good control of the inflammatory activity of the disease was observed, with a significant improvement of all the variables analysed during its evolution.

Disclosure of Interest: None declared

DOI: 10.1136/annrheumdis-2018-eular.6465

\section{AB1339 ASSOCIATION OF ANTI-THYROID ANTIBODIES AND MUSCULOSKELETAL PAIN IN PATIENTS WITH AUTOIMMUNE THYROIDITIS}

P. Rodriguez Henriquez ${ }^{1}$, R. López-Vargas ${ }^{2}$, N. Mateos-Santacruz ${ }^{3}$ ${ }^{1}$ Rheumatology; ${ }^{2}$ Internal Medicine; ${ }^{3}$ Endocrinology, Hospital General Dr. Manuel Gea González, Mexico, Mexico

Background: Autoimmune thyroiditis is closely associated with autoimmune diseases and can exhibit musculoskeletal pain as a clinical manifestation; the exact pathogenic mechanism of coexistence with other autoimmune disorders has not been clearly defined. There are very few studies that describe the association of anti-thyroid antibodies and musculoskeletal pain in patients with controlled autoimmune thyroiditis without rheumatologic disease

Objectives: To Determine the association of anti-thyroid antibodies and musculoskeletal pain in euthyroid patients with autoimmune thyroiditis who doesn't meet any criteria of a rheumatic disease

Methods: Fifty consecutive euthyroid (TSH test in a range of $0.34-5.6 \mu U / \mathrm{mL}$ and Ft4 $0.54-1.64 \mathrm{ng} / \mathrm{dL}$ ) patients with a diagnosis of autoimmune thyroiditis (positive anti peroxidase antibodies [TPOAb] $>10 \mathrm{IU} / \mathrm{mL}$ and/or anti tiroglobulin antibodies $[T G A b] \geq 5 \mathrm{IU} / \mathrm{mL}$ ), who attended the endocrinology clinic in an oneyear period were included. At the moment of enrollment the patients didn't meet any classification criteria of a rheumatic disease. The presence of musculoskeletal pain was assessed using the survey "Program of modified community orientation for rheumatic diseases" (COPCORD) phase II, validated for the Mexican population, and used as a tool to determine the prevalence of rheumatic and mus culoskeletal diseases [ $\left.{ }^{6}\right]$. COPCORD questionnaire consists of several sections such as number of affected joints, anatomic sites and pain severity measured by Visual Analogue Scale (VAS) with values ranged from 0 to 10. Descriptive statistical analyses were performed using the mean, range and standard deviation of variables. Correlations of statistical significance between groups were carried out using Spearman's rank correlation. $\mathrm{P}$ values $<0.05$ were considered to be significant.

Results: 26 patients were TPOAb positive (830.76 IU/mL $\pm 1169.33 \mathrm{IU} / \mathrm{mL}) ; 41$ patients were TGAb positive $(257.126 \mathrm{IU} / \mathrm{mL} \pm 629.344 \mathrm{IU} / \mathrm{mL})$. There was a positive correlation between the presence of TPOAb with the number of affected joints (Spearman $r=0.9618, p=0.00001$ ) and with pain intensity (Spearman $r=0.9552$, $\mathrm{p}=0.00001$ ). Similarly, there was a positive relationship between $\operatorname{TgAb}$ with the number of joints affected $(r=0.0 .7800, p=0.00001)$ and with pain intensity $(r=0.7268, \mathrm{p}=0.00001)$. 